\title{
20. \\ Mémoire sur quelques applications de la méthode inverse des tangentes.
}

(Par Mr. Barnabé Tortolini, Professeur de Mathématiques transcendantes à l'Université de Rome.)

(Extrait du tome 79. du journal arcadique de Rome.)

1. Ẃtant donné l'équation d'une courbe entre les coordonnées rectangulaires $x, y$, on pourra toujours trouver, ou exactement, ou par approximation, la longueur d'un arc correspondant aux mêmes coordonnées $x, y$. Dans le premier cas on dit que la courbe est rectifiable; dans le second cas la rectification ne pourra s'exécuter que par le moyen d'une serie, comme il arrive pour l'ellipse, et pour l'hyperbole, dont la rectification a donné sujet aux géomètres à plusieurs recherches importantes. On pourrait proposer les problème réciproque, c'est à dire: supposant que l'arc d'une courbe plane soit une fonction déterminée de l'abscisse: trouver l'équation de la courbe entre les coordonnées rectangulaires $x$ et $y$. Avant de repondre à cette question, nous répéterons la remarque déjà faite, que l'arc $s$ pourra être donné en fonction de l'abscisse, ou en termes finies, ou par approximation, et que dans la première hypothèse seulement il y aura lieu d'un genre de courbes rectifiables. Dans ce mémoire on trouvera la solution de cette double question, avec des applications choisies; on y trouvera en outre la recherche de l'équation des courbes qui sont les développées des précédentes, pourvu qu'elles soient rectifiables. Les exemples qu'on a choisis, se rapportent particulièrement aux cas dans lesquels l'arc $s$ et l'abscisse $x$ expriment une parabole d'un ordre donné $m+n$; c'est pourquoi je donne les formules qu'on trouve dans le Bulletin des sciences de Ferussac pour l'année 1825, où est dit qu'on les a extrait d'un ouvrage de M. $\boldsymbol{Q}$. $\boldsymbol{F}$. Werneburg de Jena qui a pour titre Curvarum aliquot nuper repertarum synopsis. Comme je n'ai aucune connaissance, ni de l'ouvrage indiqué, ni de la continuation des extraits qu'on en promet dans le bulletin cité, je ne manquerai pas dans la suite d'examiner les cas dans lesquels 
l'arc $s$ et l'abscisse $x$ expriment une ellipse, un cercle, une hyperbole, une hyperbole équilatère, ou enfin une cycloïde ou une logarithmique. Quoique ces recherches n'aient rien de remarquable du côté de l'analyse, elles ne seront pas cependant tout à fait denuées d'utilité si on les regarde comme un exercice pour les jeunes étudiants daus les nouvelles applications du calcul intégral à la théorie des courbes.

Soient donc $x, y$ les coordonnées rectangulaires d'un point quelconque d'une courbe plane, et l'arc $s$ soit exprimé par l'équation générale

$$
\boldsymbol{s}=\varphi(\boldsymbol{u}) \text {. }
$$

Pour tirer de cette formule l'équation entre les coordonnées $x, y$, il suffit d'observer qu'outre la formule ordinaire

$$
d s^{2}=d x^{2}+d y^{2}
$$

on a encore

$$
d s=\varphi^{\prime}(u) d x ;
$$

donc l'élinination donne

$$
d y=d x \sqrt{ }\left(\varphi^{\prime 2}(u)-1\right)
$$

et en intégrant,

$$
y=\int d x \sqrt{ }\left(\varphi^{2}(u)-1\right)+C .
$$

L'intégration sera plus ou moins facile selon la forme de $\varphi(u)$. II nous sera donc utile d'introduire l'angle formé par la droite, qui touche la courbe en un des ses points, avec l'axe des $y$ : cet angle que nous appellerons $\alpha$, sera donné par la formule

$$
d y=\cot \alpha d x
$$

qui résulte encore des autres

$$
d x=d s \sin \alpha, \quad d y=d s \cos \alpha .
$$

Mais d'ailleurs nous avons par la condition établie:

on aura donc

$$
d x=\frac{d s}{p^{\prime}(u)}
$$

$$
\varphi^{\prime}(u)=\frac{1}{\sin \alpha}=\operatorname{cosec} \alpha,
$$

et reciproquement $x$ sera fonction de $\alpha$, c'est $\dot{a}$ dire

$$
x=\psi(\operatorname{cosec} \alpha) \text {, }
$$

d'où l'on tire, en différenciant,

$$
d x=-\psi^{\prime}(\operatorname{cosec} \alpha) \frac{\cos \alpha}{\sin ^{2} \frac{\alpha}{\alpha}} d \alpha .
$$

Cette valeur étant substituée dans celle de $d y$, nous aurons, en intégrant, 


$$
y=-\int \psi^{\prime}(\operatorname{cosec} \alpha) \frac{\cos ^{2} \alpha}{\sin ^{3} \frac{\alpha}{\alpha}} d \alpha+C,
$$

et en transformant le sinus et le cosinus en cosecante,

$$
y=-\int \psi^{\prime}(\operatorname{cosec} \alpha) \operatorname{cosec} \alpha\left(\operatorname{cosec}^{2} \alpha-1\right) d \alpha+C .
$$

L'intégrale de la formule précédente, avec la valeur de $x$, conduit par la voie de l'élimination à l'équation entre les coordonnées rectangulaires $x$ et $y$. L'arc $s$ sera donné en fonction de l'angle $\alpha$ par les expressions

$$
\boldsymbol{s}=\varphi(\boldsymbol{u})=\varphi(\psi(\operatorname{cosec} \alpha))=\boldsymbol{P}(\operatorname{cosec} \alpha) \text {. }
$$

Après cela il ne sera pas difficile de déterminer le rayon $\varrho$ du cercle osculateur en partant de l'équation connue

$$
\varrho= \pm \frac{d s}{d \beta}
$$

où $\beta$ est le complément de $\alpha$. On aura donc

$$
d \beta=-d \alpha, \quad \rho=\mp \frac{d s}{d \alpha} .
$$

Or l'on tire des équatious précédentes:

$$
d s=\frac{d x}{\sin \alpha}, \quad d \alpha=-\frac{\sin ^{2} \alpha d \alpha}{\cos \alpha \psi^{\prime}(\operatorname{cosec} \alpha)},
$$

et en substituant:

$$
\rho= \pm \frac{\operatorname{cosec}^{2} \alpha \sqrt{ }\left(\operatorname{cosec}^{2} \alpha-1\right)}{\psi^{\prime}(\operatorname{cosec} \alpha)}
$$

L'expression du rayon du cercle osculateur que nous avons trouvé se réduira à une fonction de $x$ seul en éliminant l'angle $\alpha$ par le moyen des relations qui existent entre $x$ et $\alpha$. Avec la même facilité nous y parviendrons en faisant usage d'une autre expression du rayon du cercle osculateur où $d x$ est constant. Cette expression est la suivante:

$$
\rho= \pm \frac{d s^{3}}{d x d^{2} y}
$$

Or les équations déjà établies donnent

$$
d s^{3}=\left(\varphi^{\prime}(u)\right)^{3} d x^{3}, \quad d^{2} y=\frac{\varphi^{\prime}(u) \varphi^{\prime \prime}(u) d x^{2}}{\sqrt{\left(\left(\varphi^{\prime}(u)\right)^{2}-1\right.}},
$$

de manière qu'en substitant on obtient

$$
\varrho=\frac{\left(\varphi^{\prime}(u)\right)^{2}}{\varphi^{\prime \prime}(u)} \sqrt{ }\left(\varphi^{\prime}(u)\right)^{2}-1 .
$$

$2^{\circ}$. Les formules précédentes peuvent être appliquées aisément si $s$ est déterminé par les équations

$$
s^{2}=p x, \quad s=\sqrt{ }(p x)
$$


d'où, en différenciant et divisant par $d s$, on tire

et de là

$$
\frac{d x}{d s}=\frac{2 \sqrt{ }(p x)}{p}=\sin \alpha,
$$

$$
x=\frac{1}{4}\left(p \sin ^{2} \alpha\right), \quad d x=\frac{1}{2}(p \sin \alpha \cos \alpha d \alpha) \text {. }
$$

En substituant cette expression de $x$ dans celle de l'arc $s$, cette dernière devient

$$
s=\frac{1}{2} p \sin \alpha \text {. }
$$

La valeur de $d x$ substitué dans celle de $d y$, donne aussi

$$
d y=\frac{1}{2} p \cos ^{2} \alpha d \alpha,
$$

et en intégrant:

$$
y=\frac{1}{2} p \int \cos ^{2} \alpha d \alpha+C .
$$

Avant de procéder à cette intégration très-facile, j'observe que l'équation différentielle de la courbe se trouve en faisant

et l'on aura

$$
\cot \alpha=\frac{\sqrt{ }(1-\sin \alpha)}{\sin \alpha}=\frac{\sqrt{ }\left(p^{2}-4 p x\right)}{2 \sqrt{ }(p x)},
$$

$$
d y=\frac{1}{2} d x /\left(\frac{p-4 x}{x}\right)
$$

L'intégration de la première formule, exprimée par $\alpha$, est la plus facile, et on aura évidemment

$$
y=\frac{1}{4} p(\alpha+\sin \alpha \cos \alpha)+C,
$$

et comme pour $\alpha=0$ on a $y=0$, on aura $\boldsymbol{C}=0$, et $\boldsymbol{x}$ et $y$ en fonction de $\alpha$ seront

$$
y=a(2 \alpha+\sin 2 \alpha), \quad x=a(1-\cos 2 \alpha),
$$

où pour abréger on a fait $p=8 a$. Les équations trouvées représentent évidemment une cycloïde dont le diamètre est $2 a=\frac{1}{4} p$, et l'élimination de l'angle $\alpha$ nous donne l'équation connue

$$
y=\sqrt{ }\left(2 a x-x^{2}\right)+a \cdot \operatorname{arc}\left(\sin =\frac{\sqrt{ }\left(2 a x-x^{2}\right)}{a}\right) .
$$

Donc si, en partant de l'extremité supérieure de la cycloïde, on décrit une parabole dont le paramètre est le quadruple du diamètre du cercle générateur, les arcs correspondants de la cycloïde seront égaux aux ordonnées respectives de la parabole. Cette propriété ou cette rélation des deux courbes ne s'étend pas au delà du foyer de la parabole, car les ordonnées de la cycloïde deviennent imaginaires pour des valeurs de $x>2 a$ ou pour $x>\frac{1}{4} p$. Cette conséquence peut aussi se déduire en observant que l'arc $s$ d'une cycloïde qui commence a l'extremité du diamètre $2 a$ est exprimé par

$$
s=2 \sqrt{ }(2 a x) \text { ou } s^{2}=8 a x:
$$


équation d'une parabole dont le paramètre est $p=8 a$, en prenant depuis l'extremité des abscisses autant de droites égales à $2 \sqrt{ }(2 a x)$.

$3^{\circ}$. L'application faite à une parabole de second ordre peut s'étendre à une parabole de l'ordre $m+n$, et dont l'équation est

$$
s^{m+n}=p^{n} x^{m},
$$

d'où par la différenciation on a

$$
(\boldsymbol{m}+\boldsymbol{n}) \boldsymbol{s}^{\mathrm{m+n-1}} d s=m \boldsymbol{p}^{n} \boldsymbol{x}^{m-1} d x .
$$

En divisant par $d s$, et en y introduisant $\sin \alpha$, il viendra aisément:

$$
\sin \alpha=\frac{(m+n) s^{m+n-1}}{m p^{n} x^{m-1}}=\frac{(m+n) x}{m s} .
$$

En éliminant maintenant l'arc $s$ au moyen de la première équation du présent No. on trouvera les sinus et cosinus en fonction de l'abscisse, c'est à dire

$$
\begin{aligned}
& \sin \alpha=\frac{m+n}{m}\left(\frac{x}{p}\right)^{\frac{n}{m+n}} \text { et } \\
& \cos \alpha=\left[1-\left(\frac{m+n}{m}\right)^{2}\left(\frac{x}{p}\right)^{\frac{2 n}{m+n}}\right]^{\frac{1}{2}},
\end{aligned}
$$

d'où, en faisant pour abréger

$$
q=p\left(\frac{m}{m+n}\right)^{\frac{m}{n}}
$$

on tire les valeurs suivantes de $x$ et $s$ exprimées par $\alpha$ :

$$
x=\frac{m}{m+n} q(\sin \alpha)^{\frac{m+n}{n}}, \quad s=q(\sin \alpha)^{\frac{m}{n}} .
$$

Les valeurs des lignes trigonométriques sinus et cosinus déviendront fonctions de l'arc $s$ en changeant l'abscisse $x$ dans l'arc $s$ et l'exposant $\frac{n}{m+n}$ en $\frac{n}{m}$. Des équations précédentes, en prenant la valeur de la cotangente en fonction de l'abscisse $x$, on tire l'équation différentielle de la courbe:

$$
d y=\frac{\left[1-\left(\frac{m}{m+n}\right)^{n}\left(\frac{x}{p}\right)^{\frac{2 n}{m+n}}\right]^{\frac{1}{2}}}{\frac{m+n}{m}\left(\frac{x}{p}\right)^{\frac{n}{m+n}}} .
$$

Il n'est pas difficile de trouver l'équation différentielle. entre $\alpha$ et $y$, laquelle sera plus commode pour les applications; la valeur précédente de $x$, différenciée 
nous donne

et par conséquent

$$
d x=\frac{m q}{n}(\sin \alpha)^{\frac{m}{n}} \cos \alpha d \alpha
$$

ou plus simplement

$$
d y=\frac{m \eta}{n}(\sin \alpha)^{\frac{m}{n}-1} \cos ^{2} \alpha d \alpha
$$

$$
d y=q \cos \alpha d \cdot(\sin \alpha)^{\frac{m}{n}}
$$

Les formules de ce No. sont celles que l'on trouve dans le Bulletin cité de Mr. Ferussac. L'intégrale de la formule précédente, avec la valeur de $x$, représente l'équation de la courbe demandée, et l'élimination de l'angle $\alpha$ fournira l'équation aux coordonnées rectangulaires. Enfin, pour obtenir la longueur du rayon du cercle osculateur, il suffira de différencier la valeur précédente de l'arc $s$, et de diviser le tout par $d \alpha$ : il viendra

$$
\rho= \pm \frac{m q}{n}(\sin \alpha)^{\frac{m-n}{n}} \cos \alpha \text {. }
$$

$4^{\circ}$. Toutes les valeurs établies se simplifient en posant $n=1$, et en considérant ainsi une parabole de l'ordre $m+1$ dont l'équation est

Comme on a non seulement

$$
s^{m+1}=p x^{m} \text {. }
$$

$$
q=p\left(\frac{m}{1+m}\right)^{m}
$$

mais encore

$$
\begin{gathered}
s=q \sin ^{m} \alpha, \quad d s=m q \sin ^{m-1} \alpha \cos \alpha d \alpha \\
x=\frac{m}{1+m} q \sin ^{m+1} \alpha, \quad d x=m q \sin ^{m} \alpha \cos \alpha d \alpha
\end{gathered}
$$

on aura pour l'angle $\alpha$ les deux équations

$$
\sin \alpha=\frac{1+m}{m}\left(\frac{x}{p}\right)^{\frac{1}{1+m}}, \quad \cos \alpha=\left[1-\left(\frac{1+m}{m}\right)^{2}\left(\frac{x}{p}\right)^{\frac{2}{1+m}}\right]^{\frac{1}{2}},
$$

ou bien

$$
\sin \alpha=\frac{1+m}{m}\left(\frac{s}{p}\right)^{\frac{1}{m}}, \quad \cos \alpha=\left[1-\left(\frac{1+m}{m}\right)^{2}\left(\frac{s}{p}\right)^{\frac{2}{m}}\right]^{\frac{1}{2}},
$$

desquelles on tire l'équation différentielle

$$
d y=\frac{\left[1+\left(\frac{1+m}{m}\right)^{2}\left(\frac{x}{p}\right)^{\frac{2}{1+m}}\right]^{\frac{1}{2}}}{\frac{1+m}{m}\left(\frac{x}{p}\right)^{\frac{1}{1+m}}} d x
$$

Crelle's Journal f, d. M. Bd. XXVI. Heft 4. 
294 20. B. Tortolini, applications de la méthode inverse des tangentes.

ou biell celle-ci:

$$
d y=q \cos \alpha d \cdot \sin ^{m} \alpha=m q \sin ^{m-1} \alpha \cos ^{2} \alpha d \alpha ;
$$

et enfin le rayon de courbure

$$
\varrho= \pm m q \sin ^{m-1} \alpha \cos \alpha .
$$

L'intégrale trigonométrique qui exprime la valeur de l'ordonnée $y$, sera composée d'une suite de termes différents, selon que l'exposant $m$ est pair ou impair, en observant que par les artifices connus de l'intégration par parties on pourra le décomposer dans les deux termes

$$
y=q\left(\sin ^{m} \alpha \cos \alpha+\int \sin ^{m+1} \alpha d \alpha\right)+C .
$$

Faisant $m+1=\nu$, $m$ sera pair pour des valeurs impaires de $\nu$, et impair pour des valeurs paires de $\nu$, et on tronve pour des valeurs impaires de $v$ :

$\int \sin ^{\nu} \alpha d \alpha=-\frac{\cos \alpha}{\nu}\left[\sin ^{\nu-1} \alpha+\frac{\nu-1}{\nu-2} \sin ^{\nu-3} \alpha+\frac{(\nu-1)(\nu-3)}{(\nu-2)(\nu-4)} \sin ^{\nu-5} \alpha+\ldots\right.$

$$
\left.+\frac{2.4 \ldots . .(\nu-3)(\nu-1)}{1.2 .3 \ldots(\nu-4)(\nu-2)}\right]+C
$$

et pour le cas de $\nu$ pair,

$$
\begin{gathered}
\int \sin ^{\nu} \alpha d \alpha=-\frac{\cos \alpha}{\nu}\left[\sin ^{\nu-1} \alpha+\frac{\nu-1}{\nu-2} \sin ^{\nu-3} \alpha+\ldots .+\frac{3.4 \ldots(\nu-3)(\nu-1)}{2.4 \ldots(\nu-4)(\nu-2)} \sin \alpha\right] \\
+\frac{1.3 \ldots(\nu-3)(\nu-1)}{2.4 \ldots \nu-2 . \nu} \alpha+C:
\end{gathered}
$$

formules qui dans les deux cas nous fournissent le moyen de calculer les ordonnées des courbes. Passons à des applications particulières.

$5^{\circ}$. Soit $m=2$, nous aurons l'arc $s$ donné par une parabole de troisième ordre, c'est à dire par l'équation

$$
s^{3}=\mu x^{2} \text {. }
$$

Or nous avons par les formules générales:

et en outre

$$
q=\frac{4}{8} p, \quad x=\frac{2}{3} q \sin ^{3} \alpha, \quad s=q \sin ^{2} \alpha,
$$

donc, en intégrant,

$$
\begin{aligned}
& d y=2 q \sin \alpha \cos ^{2} \alpha d \alpha, \\
& y=-\frac{2}{3} q \cos ^{3} \alpha+C .
\end{aligned}
$$

Mais à $\alpha=0$ correspond $y=0$, et par conséquent la constante $C$ est $=\frac{2}{3} q$; l'intégrale complète sera douc

Enfin le rayon de courbure dévient

$$
y=\frac{2}{3} q\left(1-\cos ^{3} \alpha\right)
$$

$$
\varrho=2 q \sin \alpha \cos \alpha=q \sin 2 \alpha \text {. }
$$


Pour trouver aisément l'équation aux coordonnées rectangulaires $x, y$, faisons

$$
\frac{2}{3} q-y=y^{\prime}
$$

Effaçant l'accent, nous aurons les deux équations simultanées:

et si l'on pose

$$
x=\frac{2}{3} q \sin ^{3} \alpha, \quad y=\frac{2}{3} q \cos ^{3} \alpha,
$$

il viendra

$$
\boldsymbol{A}=\frac{2}{3} \boldsymbol{q}=\left(\frac{2}{3}\right)^{3} p
$$

$$
\left(\frac{x}{A}\right)^{\frac{1}{2}}=\sin \alpha, \quad\left(\frac{y}{A}\right)^{\frac{1}{2}}=\cos \alpha,
$$

et enfin, en élévant au carré, et sommant, nous aurons

$$
\left(\frac{x}{A}\right)^{\frac{2}{3}}+\left(\frac{y}{A}\right)^{\frac{2}{3}}=1
$$

Telle est l'équation de la courbe dont les arcs sont égaux aux ordonnées correspondantes d'une parabole cubique. Elle est évidemment semblable à la développée de l'ellipse, quoique la forme de la courbe soit tout à fait différente; car pour la développée de l'ellipse on a

$$
\left(\frac{x}{A}\right)^{\frac{2}{2}}+\left(\frac{y}{A}\right)^{\frac{2}{2}}=1
$$

et il ne peut être $A=B$ sans que $A=0=B$. En outre, comme on a évidemment

$$
\sin \alpha \cos \alpha=\left(\frac{x y}{A^{2}}\right)^{\frac{3}{3}}
$$

l'expression très simple du rayon du cercle osculatear de cette courbe est

$$
\rho=2 q\left(\frac{x y}{A^{2}}\right)^{\frac{1}{3}} \text {. }
$$

$6^{\circ}$. Supposant le nombre $m=3$, on a entre l'arc $s$ et l'abscisse $x$ l'équation d'une parabole de quatrième ordre, c'est à dire l'équation

$$
s^{4}=p x^{3}
$$

et les équations ordinaires du No. 4. nous donnent

$$
q=\left(\frac{3}{4}\right)^{3} p, \quad s=q \sin ^{3} \alpha, \quad x=\frac{3}{4} q \sin ^{4} \alpha,
$$

avec lesquelles doit coëxister l'équation suivante:

$$
d y=3 q \sin ^{2} \alpha \cos ^{2} \alpha d \alpha
$$

qui, étant integrée, donne

$$
y=-\frac{3}{8} 9 \cdot \frac{1}{4}(\sin 4 \alpha-4 \alpha)+C ;
$$

et comme à $\alpha=0$ correspond $y=0$, on aura $C=0$, et la valeur de l'ordonnée $y$ se présentera sous la forme suivante: 


$$
y=\frac{1}{8}\left(\frac{3}{4}\right)^{4} p(4 \alpha-\sin 4 \alpha)
$$

à laquelle il faut toujours réunir la valeur de $x$ trouvée après la substitution de $q$, ce qui donne

$$
x=\left(\frac{3}{4}\right)^{4} p \sin ^{4} \alpha .
$$

L'élimination de l'angle $\alpha$ s'exécute en déduisant les deux formulès

$$
\sin \alpha=\sqrt{\frac{x}{A}}, \quad \alpha=\operatorname{arc}\left(\sin =\sqrt[4]{\frac{x}{A}}\right)
$$

de la précédente; où l'on a fait pour abréger

$$
\boldsymbol{A}=\left(\frac{3}{4}\right)^{4} \boldsymbol{p} \text {. }
$$

On formera ensuite les quantités $4 \alpha$ et $\sin 4 \alpha$ pour les substituer dans la valeur de $y$. Nous pourrons cependant nous dispenser de chercher ces valeurs, et démontrer plutôt que la courbe dont il s'agit a quelque analogie avec la cycloïde. Si dans cette vue on transforme la quatrième puissance du sinus en cosinus des arcs doubles et quadruples, on obtiendra

et en supposant

$$
x=\frac{1}{8} A(3-4 \cos 2 \alpha+1+\cos 4 \alpha)
$$

nous aurons les deux équations

$$
x-\frac{1}{8} A(3-4 \cos 2 \alpha)=x^{\prime}
$$

après avoir fait

$$
x^{\prime}=a\left(1-\cos 4 \alpha^{\prime}\right), \quad y=a\left(4 \alpha^{\prime}+\sin 4 \alpha^{\prime}\right),
$$

$$
4 \alpha=180+4 \alpha^{\prime}, \quad y^{\prime}=y-180^{\prime \prime} a, \quad a=\frac{1}{8} A .
$$

Ces équations pourraient représenter l'équation d'une cycloïde, si $x^{\prime}$ n'était pas une fonction simultanée de $x$ et de l'angle $\alpha$.

7. Soit en outre, pour les applications plus nombreuses, le nombre $m=4$, on aura une parabole de cinquième ordre

$$
s^{5}=p x^{4}
$$

et les équations du No.4., dont nous avons déjà plusieurs fois fait usage, nous douneront

et en même temps

$$
q=\left(\frac{4}{5}\right)^{4} p
$$

$$
s=\left(\frac{4}{5}\right)^{4} p \sin ^{4} \alpha, \quad x=\left(\frac{4}{5}\right)^{5} p \sin ^{5} \alpha, \quad d y=4 q \sin ^{3} \alpha \cos ^{2} \alpha d \alpha,
$$

et en intégrant

$$
y={ }_{15}^{4} q \cos \alpha\left(3 \sin ^{4} \alpha-\sin ^{2} \alpha-2\right)+C .
$$

Comme pour $\alpha=0$ on a aussi $y=0$, la constante sera

et la valeur de $y$ est

$$
\boldsymbol{C}=\frac{8}{15} \boldsymbol{q}=\frac{2}{3}\left(\frac{4}{5}\right)^{5} p,
$$


20. B. Tortolini, applications de la méthode inverse des tanyentes. 297

où

$$
y=\frac{1}{3} A\left(\left(3 \sin ^{4} \alpha-\sin ^{2} \alpha-2\right) \cos \alpha+2\right),
$$

$$
\boldsymbol{A}=\left(\frac{4}{5}\right)^{5} \boldsymbol{p}
$$

On devrait encore éliminer ici l'angle $\alpha$ au moyen de l'équation

$$
\sin \alpha=\sqrt[5]{\frac{x}{A}}
$$

et former ensuite les fonctions trigonométriques correspondantes; mais au lieu d'exécuter ce calcul, nous nous bornerons à faire voir comment cette courbe adıet quelque analogie avec la courbe dont l'équation est

$$
\left(\frac{x}{A}\right)^{\frac{2}{5}}+\left(\frac{y}{A}\right)^{\frac{2}{5}}=1
$$

En effet si dans la valeur de $y$ on transforme $\sin ^{2} \alpha, \sin ^{3} \alpha, \sin ^{4} \alpha$ en $\cos \alpha$, $\cos ^{3} \alpha, \cos ^{5} \alpha$, il vient l'expression

à laquelle il faut réunir l'autre

$$
y=\frac{1}{3} A\left(2+\frac{1}{4}\left(12 \cos ^{5} \alpha-5 \cos 3 \alpha-8 \cos \alpha\right)\right)
$$

Faisant ensuite

$$
x=A \sin ^{5} \alpha
$$

$$
y-\frac{1}{3} A\left(2-\frac{1}{4}(5 \cos 3 \alpha+8 \cos \alpha)\right)=y^{\prime},
$$

on aura les deux équations très simples

$$
x=A \sin ^{5} \alpha, \quad y^{\prime}=A \cos ^{5} \alpha,
$$

par lesquelles on obtient immédiatement l'équation

$$
\left(\frac{x}{A}\right)^{\frac{2}{5}}+\left(\frac{y^{\prime}}{A}\right)^{\frac{2}{5}}=1
$$

qui représenterait une courbe comprise dans l'équation plus générale

$$
\left(\frac{x}{A}\right)^{m}+\left(\frac{y}{A}\right)^{m}=1,
$$

si $y^{\prime}$ n'était pas une fonction simultanée de $y$ et de $\alpha$. On pourrait de cette manière pousser plus loin les applications pour les paraboles des ordres supérieures, mais il sera toutefois plus utile de les supprimer, et de diriger plutôt nos recherches aux lignes de second ordre, dont nous avons dejà traité l'exemple de la parabole Apollonienue. Nous choisirons une ellipse, et nous nous proposerons de chercher une équation telle de cette courbe que ses arcs soient égaux aux ordonnées de l'ellipse des arcs $2 a, 2 b$.

$8^{\circ}$. S'il entre l'arc $s$ et l'abscisse $x$ subsiste l'équation

$$
\frac{x}{a^{2}}+\frac{s^{2}}{b^{2}}=1
$$


elle nous donne, différenciée, et divisée par $d s$, d'après les dénominations établies :

d'où

$$
\sin \alpha=-\frac{a^{2}}{b^{2}} \cdot \frac{s}{x}=-\frac{a}{b} \cdot \frac{\sqrt{ }\left(a^{2}-x^{2}\right)}{x},
$$

et ensuite

$$
x= \pm \frac{a^{2}}{\sqrt{\left(a^{2}+b^{2} \sin ^{2} \alpha\right)}}
$$

$$
s=\mp \frac{b^{2} \sin \alpha}{\sqrt{\left(a^{2}+b^{2} \sin ^{2} \alpha\right)}}
$$

La valeur de $x$, différenciée, donne

$$
d x=\mp \frac{a^{2} b^{2} \sin \alpha \cos \alpha d \alpha}{\left(a^{2}+b^{2} \sin ^{2} \alpha\right)^{\frac{3}{2}}}
$$

et par conséquent l'équation différentielle entre $x$ et $y$ devient

$$
d y=\mp \frac{a^{2} b^{2} \cos ^{2} \alpha d \alpha}{\left(a^{2}+b^{2} \sin ^{2} \alpha\right)^{\frac{3}{2}}} \text {. }
$$

Intégrant cette dernière équation, et éliminant $\alpha$ au moyen de la valeur de $x$, on aurait l'équation de la courbe entre les coordonnées rectangulaires $x$, $y$ : mais comme l'intégration ne peut s'executer que par approximation, nous déduirons des formules établies plusieurs conséquences. Faisant premièrement $a=b$, les formules précédentes se transforment en

$$
x= \pm \frac{a}{\sqrt{\left(1+\sin ^{2} \alpha\right)}}, \quad s=\mp \frac{a \sin \alpha}{\sqrt{\left(1+\sin ^{2} \alpha\right)}},
$$

et en même temps on a

$$
d y=\mp \frac{a \cos ^{2} \alpha d \alpha}{\sqrt{\left(1+\sin ^{2} \alpha\right)}}
$$

dont l'intégrale est également irréductible en termes finies, et pourrait dépendre d'une fonction elliptique de second espèce. Pour avoir quelque résultat élégant, appelons $v$ un angle qui vérifie l'équation de l'ellipse par le moyen des formules

$$
x=a \cos v, \quad y=b \sin v
$$

qui seront les équations polaires de la courbe. En les différenciant, nous aurons

$$
d x=-a \sin v d v, \quad d y=b \cos v d v \text {. }
$$

Divisant la première par la seconde, on en tire la relation connue de l'angle $\alpha$ :

d'où

$$
\sin \alpha=-\frac{a}{b} \cdot \frac{\sin v}{\cos v}
$$

$$
\cot \alpha=-\frac{\sqrt{ }\left(b^{2} \cos ^{2} v-a^{2} \sin ^{2} v\right)}{a \sin v}
$$


20. B. Tortolini, applications de la méthode inverse des tangentes. 299

Cette valeur, substituée avec celle de $d x$ dans l'équation entre $y$ et $\alpha$, donnera où l'on a fait pour abréger

$$
d y=b d v \sqrt{ }\left(1-c^{2} \sin ^{2} v\right)
$$

$$
c^{2}=\frac{a^{2}+b^{2}}{b^{2}}
$$

L'intégrale de cette formule représente une fonction elliptique de second espèce, et devra subsister pour des valeurs telles de $v$ que

et par suite

$$
1-c^{2} \sin ^{2} v>0
$$

$$
\sin v<\frac{1}{c}
$$

L'hypothèse $a=b$ nous donne

$$
\sin \alpha=-\operatorname{tang} v, \quad d y=a d v \sqrt{ }\left(1-2 \sin ^{2} v\right)=a d v \sqrt{ }(\cos 2 v) \text {. }
$$

On doit avoir $\sin v<\frac{1}{\sqrt{2}}$, ce qui correspond à $v<45^{\prime \prime}$. Enfin, comme l'angle $\alpha$ est donné en fonction de $v$, de même l'angle $v$ pourra être donné en fonction de $\alpha$, et on aura évidemment les valeurs

$$
\sin v=-\frac{b \sin \alpha}{\sqrt{\left(a^{2}+b^{2} \sin ^{2} \alpha\right)}}, \quad \cos v=\frac{a}{\sqrt{\left(a^{2}+b^{2} \sin ^{2} \alpha\right)}}
$$

qui, dans l'hypothèse $a=b$, se transforment en

$$
\sin v=-\frac{\sin \alpha}{\sqrt{\left(1+\sin ^{2} \alpha\right)}}, \quad \cos v=\frac{1}{\sqrt{\left(1+\sin ^{2} \alpha\right)}} .
$$

$9^{\circ}$. Lorsque l'arc $s$ est donné par l'équation d'une hyperbole dont l'origine est au centre, savoir par l'équation

$$
\frac{x^{2}}{a^{2}}-\frac{s^{2}}{b^{2}}=1 \text {, }
$$

en différenciant cette équation, et en la divisant par $d x$, on aura toujours

$$
\sin \alpha=\frac{a}{b} \cdot \frac{\sqrt{ }\left(x^{2}-a^{2}\right)}{x},
$$

d'où l'on tire les valeurs suivantes de $x$ et de $s$ :

$$
x=\frac{a^{2}}{\sqrt{\left(a^{2}-b^{2} \sin ^{2} \alpha\right)}}, \quad s=\frac{b^{2} \sin \alpha}{\sqrt{\left(a^{2}-b^{2} \sin ^{2} a\right)}} .
$$

Différenciant la première de ces équations; il viendra

$$
d x=\frac{a^{2} b^{2} \sin \alpha \cos \alpha d \alpha}{\left(a^{2}-b^{2} \sin ^{2} \alpha\right)^{\frac{3}{2}}}
$$

Cette valeur, substituée dans l'expression de $d y$, dont on a déjà fait usage plusieurs fois, donne

$$
d y=\frac{a^{2} b^{2} \cos ^{2} \alpha d \alpha}{\left(a^{2}-b^{2} \sin ^{2} \alpha\right)^{\frac{3}{2}}}
$$


300 20. B. Tortolini, applications de la méthode inverse des tangentes.

Cette expression ne peut être intégrée que par approximation. L'intégrale dépend d'une fonction elliptique de seconde espèce. Dans l'hypothèse d'une hyperbole équilatère on $a=b$, et par conséquent

$$
x=\frac{a}{\cos v}, \quad d x=\frac{a \sin \alpha d \alpha}{\cos ^{2} \alpha} .
$$

La valeur de $d y$ devient donc

$$
d y=\frac{a d \alpha}{\cos \alpha}
$$

Intégrant, on a

et sous une autre forme:

$$
y=\frac{1}{2} a \log \left(\frac{1+\sin \alpha}{1-\sin \alpha}\right)+C,
$$

$$
y=a \log \operatorname{tang}\left(45^{\circ}+\frac{1}{2} \alpha\right)+C .
$$

Pour trouver la constante, on doit observer que pour $\alpha=0$ on a $y=0$, et $\boldsymbol{C}=0$, et pour $\boldsymbol{y}$ on obtiendra par une transformation facile,

$$
y=\frac{1}{2} a \log \left(\frac{1+\sin \alpha}{\cos \alpha}\right)^{2}
$$

Pour éliminer l'angle $\alpha$, il faut premièrement passer des logarithmes aux nombres. On aura ainsi

et comme

$$
e^{\frac{y}{\alpha}}=\frac{1+\sin \alpha}{\cos \alpha}
$$

$$
\cos \alpha=\frac{a}{x}, \quad \sin \alpha=\frac{\sqrt{ }\left(x^{2}-a^{2}\right)}{x},
$$

en chassant les radicaux, on trouvera

$$
\left(a e^{\frac{y}{a}}-x\right)^{2}=x^{2}-a^{2},
$$

et de là évidemment

$$
x=\frac{1}{2} a\left(e^{\frac{y}{a}}+e^{-\frac{y}{a}}\right):
$$

équation de la chaînette, dont les arcs sont égaux aux coordonnées respectives d'une hyperbole équilatère. L'introduction des coordonnées polaires pourra encore dans ce cas simplifier nos équations. En effet, en appelant $v$ un angle ou une coordonuée polaire, nous remarquerons qu'on vérifie l'équation de l'hyperbole au moyen des valeurs

$$
x=\frac{a}{\cos v}, \quad s=b \operatorname{tang} v,
$$

d'où, en différenciant, on tire

$$
d x=\frac{a \sin v d v}{\cos ^{2} v}, \quad . \quad d s=\frac{b d v}{\cos ^{2} v},
$$


et en divisant on obtient

$$
\sin \alpha=\frac{a \sin v}{b}, \quad \cos \alpha=\frac{\sqrt{ }\left(b^{2}-a^{2} \sin ^{2} v\right)}{b} .
$$

Ces valeurs étant substituées dans la formule $d y=\frac{\cos \alpha}{\sin \alpha} d x$ il vient

$$
d y=\frac{d v}{\cos ^{2} v} \sqrt{ }\left(b^{2}-a^{2} \sin ^{2} v\right):
$$

équation non intégrable que par les séries. $v$ doit être un angle pour lequel $\sin v<\frac{b}{a}$, et l'hypothèse $a=b$ nous donne de nonveau la formule déjà trouvée par le seul changement de $v$ en $\alpha$. Enfin il sera utile de faire voir comment on arrive plus facilement $\dot{a}$ ce dernier résultat en faisant usage immédiatement des remarques No.2. En effet, pour l'hyperbole on a

$$
\frac{d s}{d x}=\varphi^{\prime}(u)=\frac{b}{a \sqrt{\left(x^{2}-a^{2}\right)}}
$$

la valeur de $y$ du No. cité devient

$$
d y=\frac{d x \sqrt{ }\left(b^{2}-a^{2}\left(x^{2}-a^{2}\right)\right)}{a \sqrt{ }\left(x^{2}-a^{2}\right)},
$$

et si l'on substitue $x=\frac{a}{\cos v}$, on se trouve ramené à l'équation élablie.

$10^{\circ}$. Nous n'avons considéré jusqu'ici que des équations des ligues algébriques; maintenant nous appliquerons cette théorie à plusieurs courbes trauscendantes parmi lesquelles nous choisirons la cycloïde et la logarithmique. Si pour la première on suppose l'origine des coordonnées dans le point extrème du diamètre du cercle générateur qui divise la courbe en deux parties égales et semblables, l'équation différentielle entre l'arc $s$ et l'abscisse $x$ deviendra

$$
d s=d w \sqrt{\left(\frac{2 a-x}{x}\right),}
$$

$2 a$ étant le diamètre du cercle générateur : la derivée partielle de l'arc sera donc

$$
\frac{d s}{d x}=\varphi^{\prime}(u)=\sqrt{\left(\frac{2 a-x}{x}\right)} \text {. }
$$

Cette valeur étant substituée dans celle de $x$ du No. 2., on aura aisément

$$
d y=\sqrt{ } 2 . d x \sqrt{\left(\frac{a-x}{x}\right)} \text {. }
$$

Supposant

$$
d y^{\prime}=d x \sqrt{\left(\frac{a-x}{x}\right)}
$$

cette équation représentera l'équation différentielle d'une cycloïde dont le diamètre est $a$. Donc pour décrire la courbe, dont les arcs soient égaux 
302 20. B. Tortolini, applications de la méthode inverse des tangentes.

aux ordonnées d'une cycloïde dont le diamètre est $2 a$, il suffit de décrire une autre cycloïde de diamètre $a$, c'est à dire la moitié du précédent, et de prendre en même temps les ordonnées de cette dernière inultipliées par $\sqrt{ } 2$. Nous serions parvenus aux mêmes conclusions en faisant usage des équations finies de la cycloïde; comme on le verra dans la suite.

En appelant en effet $u$ un angle compris entre les limites 0 et $\pi$, les équations de la cycloïde seront, comme on sait,

$$
x=a(1-\cos u), \quad s=a(u+\sin u),
$$

et l'élimination de l'angle $u$ donnera l'équation

$$
s=a \cdot \operatorname{arc}\left(\sin =\frac{\sqrt{ }\left(2 a x-x^{2}\right.}{a}\right)+\sqrt{ }\left(2 a x-x^{2}\right) .
$$

En différenciant les valeurs de $x$ et de $s$ et en les divisant, on aura

d'où

$$
\sin \alpha=\frac{\sin u}{1+\cos u}=\operatorname{tang} \frac{1}{2} u=\sqrt{\left(\frac{x}{2 a-x}\right)},
$$

et en différenciant,

$$
x=\frac{2 a \sin ^{2} \alpha}{1+\sin ^{2} \alpha}
$$

$$
d x=\frac{4 a \sin \alpha \cos \alpha d \alpha}{\left(1+\sin ^{2} \alpha\right)^{2}}
$$

la valeur de $d y$ deviendra donc

$$
d y=\frac{4 a \cos ^{2} \alpha d \alpha}{\left(1+\sin ^{2} \alpha\right)^{2}}
$$

ou, en transformant les puissances du sinus et du cosinus en cosinus d'un arc double, on aura pour $x$ :

et pour la valeur de $d y$ :

$$
x=\frac{2 a(1-\cos 2 \alpha)}{3-\cos 2 \alpha},
$$

$$
d y=\frac{8 a(1+\cos 2 \alpha) d \alpha}{(3-\cos 2 \alpha)^{2}}
$$

Intégrant cette dernière formule et éliminant l'angle $\alpha$, on obtient l'équation entre $x$ et $y$. Cependant on pourra exécuter l'intégration plus élégamment en introduisant l'angle $u$ donné en fonction de $\alpha$ par les équations

$$
\sin \alpha=\operatorname{tang} \frac{1}{2} u, \quad \cos \alpha d \alpha=\frac{\frac{1}{2} d u}{\cos ^{2} \frac{1}{2} u} ;
$$

la valeur de $d y$ se transformera par là en

$$
d y=2 a d u \cos \frac{1}{2} u V V(\cos u) \text {. }
$$

Cette dernière formule sera rendue intégrable en appelant $v$ un autre angle tel que l'on ait

$$
\cos u=\cos ^{2} \frac{1}{2} v \text {, }
$$


20. B. Tortolini, applications de la méthode inverse des tangentes. 303 d'où

$$
\sin \frac{1}{2} u=\frac{1}{2}\left(\gamma\left(1-\cos ^{2} \frac{1}{2} v\right)\right)=\frac{\sin \frac{1}{2} v}{\sqrt{2}}
$$

Différenciant en même temps, on aura les valeurs

$$
\cos \frac{1}{2} u d u=\frac{\cos \frac{1}{2} v d v}{\sqrt{ } 2}
$$

qui, substituées, nous donnent

En intégrant on aura

$$
d y=a \sqrt{ } 2 \cdot \cos ^{2} \frac{1}{2} v d v
$$

$$
y=\sqrt{2 \cdot \frac{1}{2}} a(v+\sin v)
$$

la constante étant zéro à cause de $v=0$ pour $y=0$. Si maintenant on remarque que pour les valeurs déjà établies on a

$$
1-\cos u=\frac{1}{2}(1-\cos v) \text {, }
$$

il sera aisé de voir qu'à l'expression de $y$ doit correspondre celle-ci :

$$
x=\frac{1}{2} a(1-\cos v) \text {. }
$$

Ces expressions représenteront les équations d'une cycloïde dont le diamètre est $a$, pourvu que l'on suppose $y=y^{\prime} \sqrt{ } 2$. En éliminant $v$ on aura donc

$$
y^{\prime}=\frac{1}{2} a \operatorname{arc}\left(\sin =\frac{\sqrt{ }\left(a x-x^{2}\right)}{\frac{1}{2} a}\right)+\left(a x-x^{2}\right) .
$$

Enfin l'intégration de $y$ par rapport à $\alpha$ nous réconduit anx résultats dejà trouvées. On aurait en effet

$$
y=4 a \int \frac{(1+\cos 2 \alpha) d \cdot 2 \alpha}{(3-\cos 2 \alpha)^{2}}
$$

En vertu des formules connues du calcul intégral:

et

$$
\int \frac{(1+\cos 2 \alpha) d .2 \alpha}{(3-\cos 2 \alpha)^{2}}=\frac{4 \sin 2 \alpha}{8(3-\cos 2 \alpha)}+\frac{4}{8} \int \frac{d .2 \alpha}{3-\cos 2 \alpha}
$$

d'où

$$
\int \frac{d .2 \alpha}{3-\cos 2 \alpha}=\frac{1}{\sqrt{8}} \operatorname{arc}\left(\sin =\frac{\sin 2 \alpha \cdot \sqrt{ } 8}{3-\cos 2 \alpha}\right)
$$

$$
y=2 a\left(\frac{\sin 2 \alpha}{3-\cos 2 \alpha}+\frac{1}{\sqrt{8}} \operatorname{arc}\left(\sin =\frac{\sin 2 \alpha \cdot \sqrt{ } 8}{3-\cos 2 \alpha}\right)\right),
$$

il devra subsister en même temps l'expression de $x$ en fonction de l'angle $\alpha$, et comme on déduit de cette dernière

$$
\sin 2 \alpha=\frac{2 \cdot \sqrt{ }(2 x}{2 a} \frac{(a-x))}{-x}, \quad \cos 2 \alpha=\frac{2 a-3 x}{2 a-x},
$$

substituant dans $y$ on en tire

$$
y=\frac{1}{2} a \sqrt{ } 2 \cdot \operatorname{arc}\left(\sin =\frac{\sqrt{ }\left(a x-x^{2}\right)}{\frac{1}{2} a}\right)+\sqrt{ } 2 \cdot \sqrt{ }\left(a x-x^{2}\right):
$$

expression tout à fait identique avec celle déjà trouvée. 
304 20. B. Tortolini, applications de la méthode inverse des tangentes.

11 $^{\circ}$. Supposant une logarithmique dont l'équation est

$$
s=a \log x
$$

on en tire

$$
\sin \alpha=\frac{x}{a},
$$

d'où

$$
x=a \sin \alpha, \quad d x=a \cos \alpha d \alpha,
$$

et en même temps

$$
\cos \alpha=\frac{\sqrt{ }\left(a^{2}-x^{2}\right)}{a} ;
$$

l'équation différentielle de la courbe sera donc

$$
d y=a \frac{\cos ^{2} \alpha}{\sin \alpha} d \alpha \text {. }
$$

En intégrant on aura

$$
y=a\left(\log \operatorname{tang} \frac{1}{2} \alpha+\cos \alpha\right)+C .
$$

Soit $b$ l'ordonnée correspondante ’̀ $\alpha=90^{\circ}$, la constante est $C=b$; donc l'intégrale complète est

$$
y=a\left(\log \operatorname{tang} \frac{1}{2} \alpha+\cos \alpha\right)+b,
$$

qu'on peut mettre aussi sous la forme

$$
y-b-a \cos \alpha=a \log \frac{\sin \alpha}{1+\cos \alpha} .
$$

Substituant ici $\sin \alpha, \cos \alpha$ en fonction de $x$ et passant des logarithmes aux nombres, on obtient

$$
e^{\frac{y-b-V\left(a^{2}-x^{2}\right)}{a}}=\frac{x}{a+\sqrt{\left(a^{2}-x^{2}\right)}}
$$

Telle est l'équation de la courbe dont les arcs sont égaux aux ordonnées correspondantes d'une logarithmique

Si l'on fait usage de l'équation

$$
s=e^{\frac{x}{a}},
$$

on a

$$
\sin \alpha=\frac{a}{e^{\frac{x}{a}}}
$$

d'où

$$
x=a \log \frac{a}{\sin \alpha}, \quad d x=-\frac{a \cos \alpha d \alpha}{\sin \alpha},
$$

et l'équation différentielle entre $y$ et $\alpha$ devient

$$
d y=-a \cot ^{2} \alpha d \alpha \text {. }
$$

L'intégrale de celle-ci est évidemment

$$
y=a(\alpha+\cot \alpha)+C \text {. }
$$


20. B. Tortolini, applications de la méthode inverse des tangentes. 305

Or pour déterminer la constante, soit $b$ l'ordonnée correspondante $\dot{a}=90$ $=\frac{1}{2} \pi$, on aura

$$
\boldsymbol{C}=\boldsymbol{b}-\frac{1}{2} \boldsymbol{a} \pi \text {. }
$$

Faisant cette substitution et supposant en outre

$$
\frac{1}{2} \pi-\alpha=\beta,
$$

on obtiendra pour intégrale complète

$$
y=a(\operatorname{tang} \beta-\beta)+b \text {. }
$$

Or la valeur précédente de $\sin \alpha$ nous donne

$$
\operatorname{tang} \beta=\frac{\sqrt{\left(e^{\frac{2 x}{a}}-a^{2}\right)}}{a} ;
$$

l'équation résultante entre $x$ et $y$ sera donc donnée par l'expression

ou bien par

$$
b-y+\sqrt{ }\left(e^{\frac{2 x}{a}}-a^{2}\right)=a \cdot \operatorname{arc}\left(\operatorname{tang}=\frac{\sqrt{ }\left(e^{\frac{2 x}{a}}-a^{2}\right)}{a}\right),
$$

$$
\sqrt{ }\left(e^{\frac{2 x}{a}}-a^{2}\right)=a \operatorname{tang}\left(\frac{b-y+\sqrt{ }\left(e^{\frac{2 x}{a}}-a^{2}\right)}{a}\right) .
$$

On pourrait d'une manière semblable étendre ces propositions à d'autres exemples. Mais sans nous occuper d'autres développements, nous croyons plus utile de procéder à la détermination des équations des courbes développantes: c'est ce qui formera le sujet des Nos. suivants de ce mémoire.

Sur les équations des développantes de plusieurs courbes planes considérées dans les 8 numeros précédents de ce mémoire.

$12^{\circ}$. Représentant par l'expression ordinaire et générale

$$
y=f(x)
$$

l'équation d'une courbe rapportée $\dot{a}$ des axes rectangulaires, et appelant $X$, $\boldsymbol{Y}$ les coordonnées du centre du cercle osculateur, $\varrho$ le rayon et $s$ l'arc de la courbe correspondante, on sait que l'on a les équations

$$
\frac{Y-y}{d x}=\frac{\varrho}{d s}, \quad \frac{X-x}{d y}=-\frac{\varrho}{d s},
$$

au moyen desquelles on parvient par l'élimination de $x, y$ à une rélation entre $\boldsymbol{X}, \boldsymbol{Y}$ qui appartient à tous les centres des cercles osculateurs, et qui sera par conséquent évidemment l'équation de la développée. Si an contraire l'équation entre $\boldsymbol{X}, \boldsymbol{Y}$ était connue, on pourrait éliminer ces coordonuées et trouver ainsi une équation entre $x, y$ qui seroit celle de la développante de la courbe représentée par l'équation donnée et de la développée de la courbe représentée par l'équation précédente. Il est essentiel de remarquer que 
toutes les développées sont des courbes rectificables, car leurs arcs sont égaux aux rayons osculateurs de la développante, ou n'en différent que d'une quantité constante. Cela posé, si l'on appelle $x, y, s$ leś coordonnées et l'arc de la développée, $\boldsymbol{X}, \boldsymbol{Y}, \boldsymbol{R}, \boldsymbol{S}$ les coordonnées, le rayon osculateur et l'arc de la développante, les formules précédentes se changeront dans les suivantes:

auxquelles il faut ajouter

$$
\frac{y-Y}{d \bar{X}}=\frac{R}{d S}, \quad \frac{x-X}{d \bar{Y}}=-\frac{R}{d S},
$$

Ein outre les directions des rayons osculateurs étant les droites qui touchent la développée, il suit qu'en appelant $\alpha$ l'angle que l'axe des $y$ forme avec une droite qui touche la courbe développée dans le point $x, y$, on devra avoir

$$
\frac{d y}{d x}=\cot \alpha=-\frac{d X}{d Y},
$$

et posant l'origine des coordonnées à l'extremité de l'arc $s$, on aura seulement

$$
R=s, \quad \frac{d Y}{d X}=-\operatorname{tang} \alpha,
$$

et en même temps

$$
\frac{d X}{d s}=\cos \alpha, \quad \frac{d Y}{d s}=-\sin \alpha .
$$

De ces valeurs on tire immédiatement les expressions des coordonnées $\boldsymbol{X}$, $\boldsymbol{Y}$, savoir

$$
\boldsymbol{Y}=y-s \cdot \cos \alpha, \quad \boldsymbol{X}=x-s \cdot \sin \alpha .
$$

Or si l'on connait les expressions de $x, y, s$ en fonctions de l'angle $\alpha$, on pourra par l'élimination de cet angle parvenir à l'équation de la courbe développante.

13 $^{\circ}$. Pour appliquer les formules précédentes à un cas très-simple, supposons que $x, y$ satisfont l'équation d'un point

On pourra prendre

$$
x=a, \quad y=b \text {. }
$$

$$
\boldsymbol{s}=\sqrt{ }\left(\boldsymbol{a}^{2}+\boldsymbol{b}^{2}\right)
$$

et les dernières équations du No. 12. donneront

d'où l'on a évidemment

$$
\boldsymbol{Y}-\boldsymbol{b}=-s \cdot \cos \alpha, \quad \boldsymbol{X}-\boldsymbol{a}=-s \cdot \sin \alpha,
$$

$$
(\boldsymbol{X}-\boldsymbol{a})^{2}+(\boldsymbol{Y}-\boldsymbol{b})^{2}=\boldsymbol{a}^{2}+\boldsymbol{b}^{2}
$$

équation d'un cercle, si l'origine des coordonnées est dans un point de la circonférence. 
20. B. Tortolini, applications de la méthode inverse des tangentes. 307

14 $^{\circ}$. Avant de passer à d'autres applications, il sera utile de remarquer que la différenciation des valeurs de $\boldsymbol{X}, \boldsymbol{Y}$ donne seulement

$$
d \boldsymbol{Y}=s \cdot \sin \alpha d \alpha, \quad d \boldsymbol{X}=-s \cdot \cos \alpha d \alpha
$$

par conséquent l'arc $S$ de la développante sera détermivé par la formule

$$
\boldsymbol{d} \boldsymbol{S}=\sqrt{ }\left(\boldsymbol{d} \boldsymbol{X}^{2}+\boldsymbol{d} \boldsymbol{Y}^{2}\right)= \pm s d \alpha
$$

qui nous apprend que le rayon du cercle osculateur de la développante est égal a l'arc correspoudant de la développée. Choisissant maintenant les équations des développantes dans lesquelles l'arc $s$ exprime une parabole de l'ordre $m+n$, c'est à dire où

$$
\boldsymbol{s}^{m+n}=\boldsymbol{p}^{n} x^{m}
$$

nous n'aurons qu'à substituer les valeurs de $x, y, s$ en fonction de l'angle $\alpha$, déterminées déjà dans le No. 3., dans les dernières équations du No. 12. 11 en résultera

$$
\boldsymbol{X}=\frac{n}{m+n} q(\sin \alpha)^{\frac{m+n}{n}}, \quad \boldsymbol{Y}=\frac{m q}{n} \int(\sin \alpha)^{\frac{m}{n}-1} \cos ^{2} \alpha d \alpha-q(\sin \alpha)^{\frac{m}{n}} \cos \alpha
$$

Cela donne aisément les différentielles correspondantes

$$
d \boldsymbol{X}=\boldsymbol{q}(\sin \alpha)^{\frac{m}{n}} \cos \alpha d \alpha, \quad d \boldsymbol{Y}=(\sin \alpha)^{\frac{m+n}{n}} d \alpha
$$

On aura donc pour l'arc $\boldsymbol{S}$ :

$$
d S=q(\sin \alpha)^{\frac{m}{n}} d \alpha
$$

où la quantité $q$ est, comme daus le No. 3.,

$$
q=p\left(\frac{m}{m+n}\right)^{\frac{m}{n}}
$$

Ces formules se simplifient encore en supposant $n=1$; alors on obtient pour la courbe développée, pour laquelle

les valeurs

$$
s^{m+1}=\boldsymbol{p} \boldsymbol{x}^{m} \text { : }
$$

$$
\boldsymbol{X}=\frac{1}{m+1} q(\sin \alpha)^{m+1}, \quad \boldsymbol{Y}=m q \int \sin ^{m-1} \cos ^{2} \alpha d \alpha-q \sin ^{m} \alpha \cos \alpha,
$$

et en même temps

$$
\boldsymbol{S}=q \int \sin ^{m} \alpha d \alpha \text {. }
$$

On tire de ces dernières ce qu'on a trouvé dans le premier exemple. En supposant $m=0$, on obtiendra les équations

$$
s=p, \quad \boldsymbol{X}=q \sin \alpha, \quad \boldsymbol{Y}=-q \cos \alpha, \quad \boldsymbol{S}=q \alpha
$$

qui renferment celle du cercle

$$
\boldsymbol{X}^{2}+\boldsymbol{Y}^{2}=q^{2}
$$

Il ne sera pas inutile de remarquer que pour $m=0$ le coëficient de $\boldsymbol{p}$ dans l'expression prend la forme indéterminée $0^{\circ}$. Pour en trouver 
dans ces cas la valeur véritable, il suffit d'observer que si pour des valeurs particulières de $y, z$ uue fonction $y^{z}$ prend la forme $0^{0}$, on en trouve la véritable valeur en calculant l'expression

$$
e^{-\frac{y^{\prime} z}{z^{\prime} y}}
$$

$y^{\prime}, z^{\prime}$ étant les dérivées de $y, z$, et $e$ la base des logarithmes hyperboliques * *). De cette manière, en faisant

$$
y=\frac{m}{m+1}, \quad z=m,
$$

la différenciatiou par rapport à $m$ donne

$$
y^{\prime}=\frac{1}{(m+1)^{2}}, \quad z^{\prime}=1,
$$

et l'exponentielle en question se change en

$$
e^{-\frac{m}{m+1}}
$$

qui pour $m=0$ sera égal à l'unité, et par conséquent $q=p$.

$15^{\circ}$. Si l'on prend pour second exemple $m=1$, on a pour la cycloïde développée les équations du No. 2., c'est à dire

$$
y=\frac{1}{8} p(2 \alpha+\sin 2 \alpha), \quad x=\frac{1}{8} p(1-\cos 2 \alpha) \quad \text { et } \quad s=\frac{1}{2} p \sin \alpha \text {; }
$$

par conséquent les équations ordinaires de la développante du No. 12. deviennent

$$
\boldsymbol{Y}=\frac{1}{8} \boldsymbol{p}(2 \alpha-\sin 2 \alpha), \quad \boldsymbol{X}=-\frac{1}{8} \boldsymbol{p}(1-\cos 2 \alpha)
$$

qui appartiennent évidemment à une cycloïde du même diamètre que la cycloïde développée; la cycloïde est donc la développée elle même.

$16^{\circ}$. Supposons $m=2$ pour la courbe développée, on aura l'arc $s^{3}=p x^{2}$, et l'équation sera

$$
\left(\frac{x}{A}\right)^{\frac{2}{5}}+\left(\frac{y^{\prime}}{A}\right)^{\frac{3}{5}}=1 \text {. }
$$

Prenant ensuite les valeurs de $x, y, s$ qu'on trouve dans le No. 5. En les substituaut dans les expressions de $\boldsymbol{X}, \boldsymbol{Y}$ dont on a déjà fait usage, on en tire pour les équations de la courbe développante:

$$
\boldsymbol{Y}=\frac{2}{3} q\left(1-\cos ^{3} \alpha\right)-q \sin ^{2} \alpha \cos \alpha, \quad \boldsymbol{X}=-\frac{1}{3} q \sin ^{3} \alpha ;
$$

d'où par l'élimination de $\alpha$ on obtient la rélation cherchée entre $X, Y$. Enfin l'arc $\boldsymbol{S}$ de cette courbe sera donné par l'expression

$$
S=q \int \sin ^{2} \alpha d \alpha=\frac{1}{4} q(2 a-\sin 2 \alpha)
$$

17 $^{\circ}$. Prenant pour équations de la développée les suivantes:

$$
x=\frac{a^{2}}{\sqrt{\left(a^{2}-b^{2} \sin ^{2} \alpha\right)}}, \quad y=a^{2} b^{2} \int \frac{\cos ^{2} \alpha d \alpha}{\sqrt{\left(a^{2}-b^{2} \sin ^{2} \alpha\right)^{3}}},
$$

*) Voir Cauchy Calcul différentiel pag. 45. Paris, 1829. 
20. B. Tortolini, applications de la méthode inverse des tangentes. 309 où l'arc $s$ exprime une hyperbole

et eu même temps

$$
\frac{x^{2}}{a^{2}}-\frac{s^{2}}{b^{2}}=1
$$

$$
s=\frac{b^{2} \sin \alpha}{\sqrt{\left(a^{2}-b^{2} \sin ^{2} \alpha\right.}}
$$

la substitution de ces valeurs donne

$$
Y=a^{2} b^{2} \int \frac{\cos ^{2} \alpha d \alpha}{\sqrt{ }\left(a^{2}-b^{2}\right.} \frac{b^{2} \sin \alpha \cos \alpha}{\left.\sin ^{2} \alpha\right)^{3}}-\frac{\left.b^{2}-b^{2} \sin ^{2} \alpha\right)}{\sqrt{\left(a^{2}\right.}} .
$$

Pour trouver la forme de l'intégrale qui appartient à $\boldsymbol{Y}$, différencions cette dernière quantité, ou bien, substituons la valeur de $s$ dans la première formule du No. 12.; cela nous donnera

et ell faisant pour abréger $\frac{b^{2}}{a^{2}}=c^{2}$,

$$
d \boldsymbol{Y}=\frac{b^{2} \sin ^{2} \alpha d \alpha}{\sqrt{\left(a^{2}-b^{2} \sin ^{2} a\right)}}
$$

$$
d Y=\frac{b^{2}}{a} \cdot \frac{\sin ^{2} \alpha d \alpha}{\sqrt{\left(1-c^{2} \sin ^{2} \alpha\right)}} .
$$

En faisant usage de la notation des fonctions elliptiques de première et de seconde espèce dont Legendre s'est servi, savoir

$$
\int \frac{d \alpha}{\sqrt{\left(1-c^{2} \sin ^{2} \alpha\right)}}=\boldsymbol{F}(c, \alpha), \quad \int d \alpha \sqrt{ }\left(1-c^{2} \sin ^{2} \alpha\right)=\boldsymbol{E}(c, \alpha),
$$

on aura l'intégrale de $d y$ par la formule

$$
\boldsymbol{Y}=\boldsymbol{a}(\boldsymbol{H}(\boldsymbol{c}, \alpha)-\boldsymbol{E}(\boldsymbol{c}, \alpha))
$$

La coëxistence de cette formule avec celle qui donne $\boldsymbol{X}$ représente l'équation de la développante de la courbe de laquelle on-tire l'équation d'une hyperbole entre l'arc et l'abscisse. Dans l'hypothèse d'une hyperbole équilatère on a $a=b$, et alors

par conséquent, outre

$$
\boldsymbol{F}(\boldsymbol{c}, \alpha)=\int \frac{d \alpha}{\cos \alpha}, \quad \boldsymbol{E}(\boldsymbol{c}, \alpha)=\int \cos \alpha d \alpha,
$$

on a aussi

$$
\begin{gathered}
X=a \cos \alpha \\
X=a\left(\frac{1}{2} \log \left(\frac{1+\sin \alpha}{1-\sin \alpha}\right)-\sin \alpha\right) .
\end{gathered}
$$

Pour éliminer l'angle $\alpha$, il suffit de remarquer que

et en même temps

$$
Y+a \sin \alpha=a \log \left(\frac{1+\sin \alpha}{\cos \alpha}\right)
$$

par conséquent

$$
\sin \alpha=\frac{\sqrt{ }\left(a^{2}-X^{2}\right)}{a},
$$

$$
\boldsymbol{Y}+\sqrt{ }\left(a^{2}-\boldsymbol{X}^{2}\right)=a \log \left(\frac{a+\sqrt{ }\left(a^{2}-X^{2}\right)}{X}\right) .
$$

Crelle's Journal f. d. M. Bd. XXVI. Heft 4. 
Telle est la développante de la chainette dont l'équation est

$$
x=\frac{1}{2} a\left(e^{\frac{y}{a}}+e^{-\frac{y}{a}}\right) \text {. }
$$

$18^{\circ}$. Cherchons enfin la développante de la courbe pour laquelle entre l'arc $s$ et l'abscisse $x$ subsiste l'équation d'une logarithmique, c'est à dire

$$
s=a \log x \text {. }
$$

Par les formules du No. 11. on a

$$
s=a \log (\sin \alpha), \quad x=a \sin \alpha, \quad y=b+a\left(\log \operatorname{tang} \frac{1}{2} \alpha+\cos \alpha\right) \text {. }
$$

La substitution de ces valeurs dans les expressions déjà obtenues de $\boldsymbol{X}, \boldsymbol{Y}$ donne

$$
\begin{gathered}
Y=b+a\left(\log \operatorname{tang} \frac{1}{2} \alpha+\cos \alpha\right)-a \cos \alpha \log (\sin \alpha), \\
\boldsymbol{X}=a \sin \alpha-a \sin \alpha \log (\sin \alpha) .
\end{gathered}
$$

L'existence de ces deux équations et l'élimination de l'angle $\alpha$ donne pour l'équation de la développante de la courbe:

$$
e^{\frac{y-b-\sqrt{ }\left(a^{2}-x^{2}\right)}{a}}=\frac{x}{a+\sqrt{ }\left(a^{2}-x^{2}\right)} .
$$

Si l'on avoit choisi la logarithmique de la forme

alors, puisque comme au No. 11.

$$
s=e^{\frac{x}{a}}
$$

$$
x=a \log \frac{a}{\sin \alpha}, \quad s=\frac{a}{\sin \alpha}, \quad y=b-\frac{1}{2}(a \pi)+a(\alpha+\cot \alpha),
$$

on auroit trouvé pour $\boldsymbol{X}$ et $\boldsymbol{Y}$ :

$$
\boldsymbol{Y}=b-\frac{1}{2}(\pi \alpha)+a \alpha, \quad X=a \log \frac{a}{\sin \alpha}-a ;
$$

et en faisant $\frac{1}{2} \pi-\alpha=\beta$, on aura plus simplement

$$
\boldsymbol{Y}=b+a \beta, \quad X=a \log \frac{a}{\cos \beta}-a .
$$

On tire de ces équations, en passant des logarithmes aux nombres,

d'où enfin

$$
e^{\frac{X+a}{a}}=\frac{a}{\cos \beta}, \quad \beta=\frac{Y-b}{a},
$$

$$
e^{\frac{x+a}{a}}=-\frac{a}{\cos \left(\frac{Y-b}{a}\right)}=a \sec \left(\frac{Y-b}{a}\right)
$$

La développée de cette courbe a pour équation

dernière formule du No. 11.

$$
\sqrt{ }\left(e^{\frac{2 x}{a}}-a^{2}\right)=a \operatorname{tang}\left(\frac{b-y+\sqrt{ }\left(e^{\frac{2 x}{a}}-a^{2}\right)}{a}\right):
$$

\section{Rome 11. Mars 1843.}

